\title{
COMMON RANDOM FIXED POINTS OF COMPATIBLE RANDOM OPERATORS
}

\author{
ISMAT BEG AND MUJAHID ABBAS
}

Received 2 June 2005; Revised 12 April 2006; Accepted 25 April 2006

We construct a random iteration scheme and study necessary conditions for its convergence to a common random fixed point of two pairs of compatible random operators satisfying Meir-Keeler type conditions in Polish spaces. Some random fixed point theorems for weakly compatible random operators under generalized contractive conditions in the framework of symmetric spaces are also proved.

Copyright @ 2006 Hindawi Publishing Corporation. All rights reserved.

\section{Introduction and preliminaries}

The study of random fixed point theory was initiated by the Prague school of probabilists in the 1950s $[12,13,26]$. Random fixed point theorems are stochastic generalization of classical fixed point theorems. The survey article by Bharucha-Reid [10] attracted the attention of several mathematicians and gave wings to this theory. Itoh [16] extended Spacek's and Hans's theorem to multivalued contraction mappings. Now this theory has become the full fledged research area and various ideas associated with random fixed point theory are used to obtain the solution of nonlinear random system (see $[9,19,25,27])$. Papageorgiou [23], Beg [3, 4], and Beg and Shahzad [6, 8] studied the structure of common random fixed points and random coincidence points of a pair of compatible random operators and proved fixed point theorems for contractive random operators in Polish spaces. Recently Beg and Shahzad [7, 8] had used different iteration processes to obtain common random fixed points. The aim of this paper is to study the necessary conditions for the convergence of random iteration scheme to common random fixed points of two pairs of compatible random operators satisfying Meir-Keeler[18] type conditions in Polish spaces. Also, in Section 3, we establish the existence of unique common random fixed points of random operators under generalized contractive conditions. We first review the following concepts which are essential for our study in this paper.

Throughout this paper, $(\Omega, \Sigma)$ denotes a measurable space ( $\Sigma$-sigma algebra). A symmetric on a set $X$ is a nonnegative real-valued function $d$ on $X \times X$ such that for all $x, y \in X$ we have

\footnotetext{
Hindawi Publishing Corporation

International Journal of Mathematics and Mathematical Sciences

Volume 2006, Article ID 23486, Pages 1-15

DOI 10.1155/IJMMS/2006/23486
} 
2 Common random fixed points of compatible random operators

(a) $d(x, y)=0$ if and only if $x=y$, and

(b) $d(x, y)=d(y, x)$.

Let $d$ be a symmetric on a set $X$. For $\varepsilon>0$ and $x \in X, B(x, \varepsilon)$ denotes the spherical ball centred at $x$ with radius $\varepsilon$, defined as the set $\{y \in X: d(y, x)<\varepsilon\}$. A topology $t(d)$ on $X$ is given by $U \in t(d)$ if and only if for each $x \in U, B(x, \varepsilon) \subset U$ for some $\varepsilon>0$. Note that $\lim _{n \rightarrow \infty} d\left(x_{n}, x\right)=0$ if and only if $x_{n} \rightarrow x$ in the topology $t(d)$. Let $F$ be a subset of $X$. A mapping $\xi: \Omega \rightarrow X$ is measurable if $\xi^{-1}(U) \in \Sigma$ for each open subset $U$ of $X$. The mapping $T: \Omega \times F \rightarrow F$ is a random map if and only if for each fixed $x \in F$, the mapping $T(\cdot, x): \Omega \rightarrow F$ is measurable. The mapping $T$ is continuous if for each $\omega \in \Omega$, the mapping $T(\omega, \cdot): F \rightarrow X$ is continuous. A measurable mapping $\xi: \Omega \rightarrow X$ is a random fixed point of random map $T: \Omega \times F \rightarrow X$ if and only if $T(\omega, \xi(\omega))=\xi(\omega)$ for each $\omega \in \Omega$. We denote the set of random fixed points of a random map $T$ by $R F(T)$ and the set of all measurable mappings from $\Omega$ into a symmetric space $X$ by $M(\Omega, X)$. We denote the $n$th iterate $T(\omega, T(\omega, T(\omega, \ldots, T(\omega, x))), \ldots)$ of $T$ by $T^{n}(\omega, x)$. The letter $I$ denotes the random mapping $I: \Omega \times F \rightarrow F$ defined by $I(\omega, x)=x$ and $T^{0}=I$. Let $\phi: \mathbb{R}^{+} \rightarrow \mathbb{R}^{+}$be a function satisfying the condition $0<\phi(t)<t$, for each $t>0$.

Definition 1.1. Let $X$ be a Polish space, that is, a separable complete metric space. Mappings $f, g: X \rightarrow X$ are compatible if $\lim _{n \rightarrow \infty} d\left(f g\left(x_{n}\right), g f\left(x_{n}\right)\right)=0$, provided that $\lim _{n \rightarrow \infty} f\left(x_{n}\right)$ and $\lim _{n \rightarrow \infty} g\left(x_{n}\right)$ exist in $X$ and $\lim _{n \rightarrow \infty} f\left(x_{n}\right)=\lim _{n \rightarrow \infty} g\left(x_{n}\right)$. Random operators $S, T: \Omega \times X \rightarrow X$ are compatible if $S(\omega, \cdot)$ and $T(\omega, \cdot)$ are compatible for each $\omega \in \Omega$. (See Beg and Shahzad [6].)

Definition 1.2. Let $X$ be a Polish space. Random operators $S, T: \Omega \times X \rightarrow X$ are weakly compatible if $T(\omega, \xi(\omega))=S(\omega, \xi(\omega))$, for some $\xi \in M(\Omega, X)$, then $T(\omega, S(\omega, \xi(\omega)))=$ $S(\omega, T(\omega, \xi(\omega)))$ for every $\omega \in \Omega$.

Definition 1.3 [29]. Let $\left\{x_{n}\right\}$ and $\left\{y_{n}\right\}$ be two sequences in symmetric space $(X, d)$ and $x, y \in X$. The space is said to satisfy the following axioms.

(w.1) $\lim _{n \rightarrow \infty} d\left(x_{n}, x\right)=\lim _{n \rightarrow \infty} d\left(x_{n}, y\right)=0$ implies that $x=y$.

(w.2) $\lim _{n \rightarrow \infty} d\left(x_{n}, x\right)=\lim _{n \rightarrow \infty} d\left(x_{n}, y_{n}\right)=0$ implies that $\lim _{n \rightarrow \infty} d\left(y_{n}, x\right)=0$.

If $X=\mathbb{R}$ is taken, define the symmetric function as $d(x, y)=e^{|x-y|}-1$ with sequences $\left\{x_{n}\right\}=\{1+1 / n\},\left\{y_{n}\right\}=\{1-1 / n\}$, and $x=1$ in $X$, then it is easy to verify that axiom (w.2) holds.

Definition 1.4. Let $\left\{x_{n}\right\}$ and $\left\{y_{n}\right\}$ be two sequences in a symmetric space $(X, d)$ and $x \in X$. The space $X$ is said to satisfy axiom $\left(H_{E}\right)$; if $\lim _{n \rightarrow \infty} d\left(x_{n}, x\right)=\lim _{n \rightarrow \infty} d\left(y_{n}, x\right)=0$ implies that $\lim _{n \rightarrow \infty} d\left(x_{n}, y_{n}\right)=0$.

For example, let $X=[0, \infty)$ with the symmetric function $d(x, y)=e^{|x-y|}-1$, then $(X, d)$ satisfies the axiom $\left(H_{E}\right)$.

Definition 1.5. Let $d$ be a symmetric function on $X$. Two random mappings $S$ and $T$ from $\Omega \times X$ to $X$ are said to satisfy property $(I)$ if there exists a sequence $\left\{\xi_{n}\right\}$ in $M(\Omega, X)$ such that for some $\xi \in M(\Omega, X)$,

$$
\lim _{n \rightarrow \infty} d\left(T\left(\omega, \xi_{n}(\omega), \xi(\omega)\right)\right)=\lim _{n \rightarrow \infty} d\left(S\left(\omega, \xi_{n}(\omega), \xi(\omega)\right)\right)=0
$$

for every $\omega \in \Omega$. 
Example 1.6. Let $\Omega=[0,1]$ and let $\Sigma$ be the sigma algebra of Lebesgue's measurable subsets of $[0,1]$. Take $X=\mathbb{R}$ with $d(x, y)=e^{|x-y|}-1$, obviously $d$ is symmetric on $\mathbb{R}$. Define two random mappings $T$ and $S$ from $\Omega \times X$ to $X$ as

$$
\begin{aligned}
& T(\omega, x)= \begin{cases}1+2 x-\omega^{2} & \text { if }(\omega, x) \in[0,1] \times[0,1], \\
3\left(1-\omega^{2}\right) & \text { otherwise, }\end{cases} \\
& S(\omega, x)= \begin{cases}\omega^{2}+4 x-1 & \text { if }(\omega, x) \in[0,1] \times[0,1], \\
3\left(1-\omega^{2}\right) & \text { otherwise. }\end{cases}
\end{aligned}
$$

Also, the sequence of measurable mappings $\xi_{n}: \Omega \rightarrow X$ is given by $\xi_{n}(\omega)=((n-1) / n)-$ $\omega^{2}$ for every $\omega \in \Omega$ and $n \in \mathbb{N}$. Define measurable function $\xi: \Omega \rightarrow X$ as $\xi(\omega)=3\left(1-\omega^{2}\right)$ for every $\omega \in \Omega$. Consider the case, when $\xi_{n}(\omega) \in[0,1]$ for every $n \in \mathbb{N}$, and $\omega \in \Omega$, then we have

$$
\begin{aligned}
\lim _{n \rightarrow \infty} d\left(T\left(\omega, \xi_{n}(\omega)\right), \xi(\omega)\right) & =\lim _{n \rightarrow \infty} e^{\left|T\left(\omega, \xi_{n}(\omega)\right)-\xi(\omega)\right|}-1 \\
& =\lim _{n \rightarrow \infty} e^{|-2 / n|}-1=0 \quad \text { for every } \omega \in \Omega, \\
\lim _{n \rightarrow \infty} d\left(S\left(\omega, \xi_{n}(\omega)\right), \xi(\omega)\right) & =\lim _{n \rightarrow \infty} e^{\left|S\left(\omega, \xi_{n}(\omega)\right)-\xi(\omega)\right|}-1 \\
& =\lim _{n \rightarrow \infty} e^{|-4 / n|}-1=0 \quad \text { for every } \omega \in \Omega .
\end{aligned}
$$

When $\xi_{n}(\omega) \notin[0,1]$ for $n \in \mathbb{N}$ and $\omega \in \Omega$, then obviously $\lim _{n \rightarrow \infty} d\left(T\left(\omega, \xi_{n}(\omega)\right), \xi(\omega)\right)=$ $\lim _{n \rightarrow \infty} d\left(S\left(\omega, \xi_{n}(\omega)\right), \xi(\omega)\right)=0$ for every $\omega \in \Omega$. So $T$ and $S$ satisfy property $(I)$.

Definition 1.7. Let $X$ be a Polish space and let $A, B, S, T: \Omega \times X \rightarrow X$ be four random operators with $A(\omega, X) \subset T(\omega, X)$ and $B(\omega, X) \subset S(\omega, X)$ for every $\omega \in \Omega$. Consider the sequence of functions $\left\{\xi_{n}\right\}$ and $\left\{\eta_{n}\right\}$ from $\Omega$ to $X$ defined by

$$
\begin{gathered}
\xi_{2 n-1}(\omega)=T\left(\omega, \eta_{2 n-1}(\omega)\right)=A\left(\omega, \eta_{2 n-2}(\omega)\right), \\
\xi_{2 n}(\omega)=S\left(\omega, \eta_{2 n}(\omega)\right)=B\left(\omega, \eta_{2 n-1}(\omega)\right),
\end{gathered}
$$

where $\xi_{0}: \Omega \rightarrow X$ is an arbitrary fixed map, define $M: X \times X \times \Omega \rightarrow \mathbb{R}^{+}$as

$$
\begin{aligned}
M(x, y, \omega)=\max \left\{d(S(\omega, x), T(\omega, y)), d(S(\omega, x), A(\omega, x)), d(T(\omega, y), B(\omega, y)), \frac{1}{2}\right. \\
{[d(S(\omega, x), B(\omega, y)),+d(T(\omega, y), A(\omega, x))]\} . }
\end{aligned}
$$


Remark 1.8. Let $F$ be a closed subset of a Polish space $X$ and let the sequence of measurable functions $\left\{\xi_{n}\right\}$ defined from $\Omega$ to $F$ be pointwise convergent, that is, $\xi_{n}(\omega) \rightarrow q:=$ $\xi(\omega)$, for each $\omega \in \Omega$. Now the closedness of $F$ implies that $\xi$ is a mapping from $\Omega$ to $F$, and $\xi$ being the limit of the sequence of measurable functions is also measurable. Since $F$ is a subset of a separable metric space $X$, so if $T$ is a continuous random operator, then by [2, Lemma 8.2.3], the map $\omega \rightarrow T^{n}(\omega, f(\omega))$ is a measurable function for any measurable function $f$ from $\Omega$ to $X$ (see also $[15,28]$ ).

\section{Common random fixed points of random operators}

Meir and Keeler [18] obtained a remarkable generalization of the Banach contraction principle (see [1, 11]). Park and Bae [24] extended Meir and Keelar's theorem for two commuting maps following Jungck's method [17]. Recent work of Pant [22] contains common fixed point theorem for four maps satisfying certain contractive conditions (also see $[5,18,20,21]$ and references mentioned therein). Beg and Shahzad [7] studied random fixed point theorems for contractive type random operators on Banach spaces. In continuation of these results now we obtain common random fixed point for four random operators under a $(\varepsilon, \delta)$ contractive condition which was initiated by Meir and Keeler [18]. Our result is a stochastic analog of a result of Pant [22]. To establish our main result, we first prove the following lemma.

Lemma 2.1. Let $X$ be a Polish space and let $A, B, S, T: \Omega \times X \rightarrow X$ be four random operators with $A(\omega, X) \subset T(\omega, X)$ and $B(\omega, X) \subset S(\omega, X)$ for every $\omega \in \Omega$. If for any given $\varepsilon>0$, there exists a $\delta>0$ such that for all $x, y \in X$,

$$
\varepsilon \leq M(x, y, \omega)<\delta+\varepsilon \Longrightarrow d(A(\omega, x), B(\omega, y))<\varepsilon \quad \text { for each } \omega \in \Omega,
$$

then, for the sequence of function $\left\{\xi_{n}\right\}$, as taken in Definition 1.7,

(a) $\lim _{n \rightarrow \infty} d\left(\xi_{n}(\omega), \xi_{n+1}(\omega)\right)=0$, for each $\omega \in \Omega$;

(b) there exists $n_{0} \in \mathbb{N}$ such that $p, q \geq n_{0}$, where $p$ and $q$ are of opposite parity, give the following implication:

$$
\varepsilon \leq d\left(\xi_{p}(\omega), \xi_{q}(\omega)\right)<\varepsilon+r \Longrightarrow d\left(\xi_{p+1}(\omega), \xi_{q+1}(\omega)\right)<\varepsilon
$$

for each $\omega \in \Omega$, and where $r=\min \{\varepsilon / 2, \delta / 2\}$;

(c) the sequence $\left\{\xi_{n}(\omega)\right\}$ is a Cauchy sequence for each $\omega \in \Omega$.

$\operatorname{Proof}$ (part (a)). It is obvious that if for every $\omega \in \Omega$ and $x, y \in X$, we take $M(x, y, \omega)=0$, then $d(A(\omega, x), B(\omega, y))=0$ for every $\omega \in \Omega$ and $x, y \in X$. So

Now, $M(x, y, \omega)>0 \Longrightarrow d(A(\omega, x), B(\omega, y))<M(x, y, \omega)$ for each $\omega \in \Omega$ and $x, y \in X$.

$$
d(A(\omega, x), B(\omega, y)) \leq M(x, y, \omega) \quad \text { for each } \omega \in \Omega, x, y \in X .
$$




\section{Consider}

$$
\begin{aligned}
& d\left(\xi_{2 n}(\omega), \xi_{2 n+1}(\omega)\right)=d\left(B\left(\omega, \eta_{2 n-1}(\omega)\right), A\left(\omega, \eta_{2 n}(\omega)\right)\right) \\
& =d\left(A\left(\omega, \eta_{2 n}(\omega)\right), B\left(\omega, \eta_{2 n-1}(\omega)\right)\right) \\
& \leq M\left(\eta_{2 n}(\omega), \eta_{2 n-1}(\omega), \omega\right) \\
& =\max \left\{d\left(S\left(\omega, \eta_{2 n}(\omega)\right), T\left(\omega, \eta_{2 n-1}(\omega)\right)\right), d\left(S\left(\omega, \eta_{2 n}(\omega)\right),\right.\right. \\
& \left.A\left(\omega, \eta_{2 n}(\omega)\right)\right), d\left(T\left(\omega, \eta_{2 n-1}(\omega)\right), B\left(\omega, \eta_{2 n-1}(\omega)\right)\right), \\
& \frac{1}{2}\left[d\left(S\left(\omega, \eta_{2 n}(\omega)\right), B\left(\omega, \eta_{2 n-1}(\omega)\right)\right)+d\left(T\left(\omega, \eta_{2 n-1}(\omega)\right),\right.\right. \\
& \left.\left.\left.A\left(\omega, \eta_{2 n}(\omega)\right)\right)\right]\right\} \\
& =\max \left\{d\left(\xi_{2 n}(\omega), \xi_{2 n-1}(\omega)\right), d\left(\xi_{2 n}(\omega), \xi_{2 n+1}(\omega)\right),\right. \\
& d\left(\xi_{2 n-1}(\omega), \xi_{2 n}(\omega)\right), \frac{1}{2}\left[d\left(\xi_{2 n}(\omega), \xi_{2 n}(\omega)\right)\right. \\
& \left.\left.+d\left(\xi_{2 n-1}(\omega), \xi_{2 n+1}(\omega)\right)\right]\right\} \\
& \leq \max \left\{d\left(\xi_{2 n}(\omega), \xi_{2 n-1}(\omega)\right), d\left(\xi_{2 n}(\omega), \xi_{2 n+1}(\omega)\right),\right. \\
& \left.\frac{1}{2}\left[d\left(\xi_{2 n-1}(\omega), \xi_{2 n}(\omega)\right)+d\left(\xi_{2 n}(\omega), \xi_{2 n+1}(\omega)\right)\right]\right\} \\
& \leq \max \left\{d\left(\xi_{2 n}(\omega), \xi_{2 n-1}(\omega)\right), d\left(\xi_{2 n}(\omega), \xi_{2 n+1}(\omega)\right)\right\}
\end{aligned}
$$

for each $\omega \in \Omega$. Now, if $M\left(\eta_{2 n}(\omega), \eta_{2 n-1}(\omega), \omega\right)=0$, then $d\left(\xi_{2 n}(\omega), \xi_{2 n+1}(\omega)\right)=d\left(\xi_{2 n}(\omega)\right.$, $\left.\xi_{2 n-1}(\omega)\right)=0$ for each $\omega \in \Omega$. But if $M\left(\eta_{2 n}(\omega), \eta_{2 n-1}(\omega), \omega\right)>0$ for every $\omega \in \Omega$, then

$$
\begin{aligned}
d\left(\xi_{2 n}(\omega), \xi_{2 n+1}(\omega)\right) & <M\left(\eta_{2 n}(\omega), \eta_{2 n-1}(\omega), \omega\right) \\
& \leq \max \left\{d\left(\xi_{2 n}(\omega), \xi_{2 n-1}(\omega)\right), d\left(\xi_{2 n}(\omega), \xi_{2 n+1}(\omega)\right)\right\}
\end{aligned}
$$

for each $\omega \in \Omega$. That is, $d\left(\xi_{2 n}(\omega), \xi_{2 n+1}(\omega)\right)<d\left(\xi_{2 n}(\omega), \xi_{2 n-1}(\omega)\right)$ for each $\omega \in \Omega$ and $n \in$ $\mathbb{N}$. Thus in any case we have

$$
\begin{aligned}
d\left(\xi_{2 n}(\omega), \xi_{2 n+1}(\omega)\right) & \leq M\left(\eta_{2 n}(\omega), \eta_{2 n-1}(\omega), \omega\right) \\
& \leq d\left(\xi_{2 n}(\omega), \xi_{2 n-1}(\omega)\right) \text { for each } \omega \in \Omega .
\end{aligned}
$$

Similarly,

$$
\begin{aligned}
d\left(\xi_{2 n+1}(\omega), \xi_{2 n+2}(\omega)\right) & \leq M\left(\eta_{2 n+1}(\omega), \eta_{2 n}(\omega), \omega\right) \\
& \leq d\left(\xi_{2 n}(\omega), \xi_{2 n+1}(\omega)\right) \text { for each } \omega \in \Omega .
\end{aligned}
$$

Thus, $\left\{d\left(\xi_{n}(\omega), \xi_{n+1}(\omega)\right)\right\}$ is nonincreasing and bounded below by 0 for each $\omega \in \Omega$. So, $\lim _{n \rightarrow \infty} d\left(\xi_{n}(\omega), \xi_{n+1}(\omega)\right)=L$ for each $\omega \in \Omega$. Now $L=0$ proves the result. Suppose $L>0$, 
6 Common random fixed points of compatible random operators

then for some $m \in \mathbb{N}$, we have

$$
\begin{aligned}
L & \leq d\left(\xi_{2 m+1}(\omega), \xi_{2 m+2}(\omega)\right) \leq M\left(\eta_{2 m+1}(\omega), \eta_{2 m}(\omega), \omega\right) \\
& \leq d\left(\xi_{2 m}(\omega), \xi_{2 m+1}(\omega)\right)<L+\delta \quad \text { for each } \omega \in \Omega .
\end{aligned}
$$

So, we have

$$
d\left(\xi_{2 m}(\omega), \xi_{2 m+1}(\omega)\right)=d\left(B\left(\omega, \eta_{2 m-1}(\omega)\right), A\left(\omega, \eta_{2 m}(\omega)\right)\right)<L \quad \text { for some } m \in \mathbb{N} .
$$

Which contradicts the choice of $L$ and this completes the proof of part (a).

Proof (part (b)). Since $\lim _{n \rightarrow \infty} d\left(\xi_{n}(\omega), \xi_{n+1}(\omega)\right)=0$ for each $\omega \in \Omega$, so, there exists a positive integer $n_{0}$ such that $d\left(\xi_{n}(\omega), \xi_{n+1}(\omega)\right)<r / 2$ for all $n \geq n_{0}$ for every $\omega \in \Omega$. Let $p, q \in \mathbb{N}$ such that $p, q \geq n_{0}$ with $p=2 n, q=2 m-1$. Suppose for each $\omega \in \Omega$,

$$
\varepsilon \leq d\left(\xi_{p}(\omega), \xi_{q}(\omega)\right)=d\left(\xi_{2 n}(\omega), \xi_{2 m-1}(\omega)\right)<\varepsilon+r .
$$

Consider

$$
\begin{aligned}
& \varepsilon \leq d\left(\xi_{p}(\omega), \xi_{q}(\omega)\right) \\
&=d\left(S\left(\omega, \eta_{2 n}(\omega)\right), T\left(\omega, \eta_{2 m-1}(\omega)\right)\right) \leq M\left(\eta_{2 n}(\omega), \eta_{2 m-1}(\omega), \omega\right) \\
&=\max \left\{d\left(S\left(\omega, \eta_{2 n}(\omega)\right), T\left(\omega, \eta_{2 m-1}(\omega)\right)\right), d\left(S\left(\omega, \eta_{2 n}(\omega)\right), A\left(\omega, \eta_{2 n}(\omega)\right)\right),\right. \\
& d\left(T\left(\omega, \eta_{2 m-1}(\omega)\right), B\left(\omega, \eta_{2 m-1}(\omega)\right)\right), \\
& \frac{1}{2}\left[d\left(S\left(\omega, \eta_{2 n}(\omega)\right), B\left(\omega, \eta_{2 m-1}(\omega)\right)\right)\right. \\
&\left.\left.+d\left(T\left(\omega, \eta_{2 m-1}(\omega)\right), A\left(\omega, \eta_{2 n}(\omega)\right)\right)\right]\right\} \\
&=\max \left\{d\left(\xi_{p}(\omega), \xi_{q}(\omega)\right), d\left(\xi_{p}(\omega), \xi_{p+1}(\omega)\right), d\left(\xi_{q}(\omega), \xi_{q+1}(\omega)\right),\right.\left.\frac{1}{2}\left[d\left(\xi_{p}(\omega), \xi_{q+1}(\omega)\right)+d\left(\xi_{q}(\omega), \xi_{p+1}(\omega)\right)\right]\right\} \\
& \leq \max \left\{d\left(\xi_{p}(\omega), \xi_{q}(\omega)\right), \frac{1}{2}\left[2 d\left(\xi_{p}(\omega), \xi_{q}(\omega)\right)+d\left(\xi_{q}(\omega), \xi_{q+1}(\omega)\right)\right.\right. \\
&\left.\left.+d\left(\xi_{p}(\omega), \xi_{p+1}(\omega)\right)\right]\right\} \\
& \leq d\left(\xi_{p}(\omega), \xi_{q}(\omega)\right)+\frac{r}{2}<\varepsilon+\frac{3 r}{2}<\varepsilon+2 r \leq \delta+\varepsilon \quad \text { for each } \omega \in \Omega .
\end{aligned}
$$

Thus,

$$
\begin{aligned}
d\left(\xi_{p+1}(\omega), \xi_{q+1}(\omega)\right) & =d\left(\xi_{2 n+1}(\omega), \xi_{2 m}(\omega)\right)=d\left(A\left(\omega, \eta_{2 n}(\omega)\right), B\left(\omega, \eta_{2 m-1}(\omega)\right)\right) \\
& <\varepsilon \quad \text { for each } \omega \in \Omega,
\end{aligned}
$$

which is required. 
Proof (part (c)). Take $\alpha=2 \varepsilon$, part (b) assures the existence of positive integer $n_{1}$ such that whenever $p, q$ are some positive integers of opposite parity and $p, q>n_{1}$, then

$$
d\left(\xi_{p+1}(\omega), \xi_{q+1}(\omega)\right)<\varepsilon \quad \text { if } \varepsilon \leq d\left(\xi_{p}(\omega), \xi_{q}(\omega)\right)<\varepsilon+r
$$

for each $\omega \in \Omega$. Since $\lim _{n \rightarrow \infty} d\left(\xi_{n}(\omega), \xi_{n+1}(\omega)\right)=0$ for each $\omega \in \Omega$, choose a positive integer $n_{0}$ such that $n_{0}>n_{1}$ and

$$
d\left(\xi_{m}(\omega), \xi_{m+1}(\omega)\right)<\frac{r}{6} \quad \text { for every } m \geq n_{0} \text { and for each } \omega \in \Omega
$$

Select $q>p \geq n_{0}$ so that (2.13) and (2.14) hold. Now we show that for each $\omega \in \Omega$, we have $d\left(\xi_{p}(\omega), \xi_{q}(\omega)\right)<\alpha$. If it is not true then for some $\omega \in \Omega$ we have

$$
d\left(\xi_{p}(\omega), \xi_{q}(\omega)\right) \geq \alpha=2 \varepsilon
$$

We first want to choose $m>p$ such that for all those $\omega \in \Omega$ for which (2.15) holds, we have the following inequality:

$$
\varepsilon+\frac{r}{3}<d\left(\xi_{p}(\omega), \xi_{m}(\omega)\right)<\varepsilon+r
$$

with $p$ and $m$ of opposite parity. Let $k$ be the smallest integer greater than $p$ such that $d\left(\xi_{p}(\omega), \xi_{k}(\omega)>\varepsilon+r / 2\right.$. Since $r<\varepsilon$, the integer $k$ exists from (2.15). Moreover, we have

$$
d\left(\xi_{p}(\omega), \xi_{k}(\omega)\right)<\varepsilon+\frac{2 r}{3}
$$

For otherwise,

$$
\begin{aligned}
\varepsilon+\frac{2 r}{3} & \leq d\left(\xi_{p}(\omega), \xi_{k}(\omega)\right) \\
& \leq d\left(\xi_{p}(\omega), \xi_{k-1}(\omega)\right)+d\left(\xi_{k-1}(\omega), \xi_{k}(\omega)\right) \\
& <d\left(\xi_{p}(\omega), \xi_{k-1}(\omega)\right)+\frac{r}{6}
\end{aligned}
$$

which gives $\varepsilon+r / 2<d\left(\xi_{p}(\omega), \xi_{k-1}(\omega)\right)$. Since $k-1>p \geq n_{0}>n_{1}$, so this contradicts the choice of $k$. Thus we have

$$
\varepsilon+\frac{r}{3}<d\left(\xi_{p}(\omega), \xi_{m}(\omega)\right)<\varepsilon+r
$$

If $p$ and $k$ are of opposite parity, we take $m=k$ in (2.19) to obtain (2.16). If $p$ and $k$ are of like parity, then $p$ and $k+1$ will be of opposite parity. Since $d\left(\xi_{k}(\omega), \xi_{k+1}(\omega)\right)<r / 6$, now, using triangle inequality, we have

$$
\varepsilon+\frac{r}{3}<d\left(\xi_{p}(\omega), \xi_{k+1}(\omega)\right)<\varepsilon+\frac{5 r}{6}
$$


8 Common random fixed points of compatible random operators

in this case, take $m=k+1$. So we can choose $m$ so that (2.16) holds. Now for $p, m \geq n_{0}$, we get

$$
\begin{aligned}
\varepsilon+\frac{r}{3} & <d\left(\xi_{p}(\omega), \xi_{m}(\omega)\right) \\
& \leq d\left(\xi_{p}(\omega), \xi_{p+1}(\omega)\right)+d\left(\xi_{p+1}(\omega), \xi_{m+1}(\omega)\right)+d\left(\xi_{m+1}(\omega), \xi_{m}(\omega)\right),
\end{aligned}
$$

which gives $\varepsilon+r / 3<r / 3+d\left(\xi_{p+1}(\omega), \xi_{m+1}(\omega)\right)<\varepsilon+r / 3$. This contradiction concludes the required result.

Theorem 2.2. Let $X$ be a Polish space and let $(A, S)$ and $(B, T)$ be two pairs of compatible random operators from $\Omega \times X$ to $X$ with $A(\omega, X) \subset T(\omega, X)$ and $B(\omega, X) \subset S(\omega, X)$ for every $\omega \in \Omega$, and

(1) for any given $\varepsilon>0$, there exists $\delta>0$ such that for all $x, y \in X$,

$$
\begin{aligned}
\varepsilon & \leq \max \{d(S(\omega, x), T(\omega, y)), d(A(\omega, x), S(\omega, x)), d(B(\omega, y), T(\omega, y))\} \\
& <\delta+\varepsilon \Longrightarrow d(A(\omega, x), B(\omega, y))<\varepsilon,
\end{aligned}
$$

for each $\omega \in \Omega$;

(2) also $d(A(\omega, x), B(\omega, y)) \leq k(\omega) d(S(\omega, x), T(\omega, y))$ for each $\omega \in \Omega$ and $x, y \in X$, where $k: \Omega \rightarrow[1, \infty)$ is a measurable mapping.

If one of the random operators $A, B, S$, or $T$ is continuous then $A, B, S$, and $T$ have unique common random fixed point.

Proof. Consider, the sequence of function $\left\{\xi_{n}\right\}$ as taken in Definition 1.7. Now contractive condition (1) in this theorem gives the similar contractive conditions as given in Lemma 2.1, so $\left\{\xi_{n}(\omega)\right\}$ is a Cauchy sequence for each $\omega \in \Omega$. Therefore, $\xi_{n}(\omega) \rightarrow \xi(\omega)$, where $\xi: \Omega \rightarrow X$, being the limit of the sequence of measurable mappings, is a measurable mapping. Now

$$
\begin{gathered}
\xi_{2 n-1}(\omega)=T\left(\omega, \eta_{2 n-1}(\omega)\right)=A\left(\omega, \eta_{2 n-2}(\omega)\right) \longrightarrow \xi(\omega), \\
\xi_{2 n}(\omega)=B\left(\omega, \eta_{2 n-1}(\omega)\right)=S\left(\omega, \eta_{2 n}(\omega)\right) \longrightarrow \xi(\omega)
\end{gathered}
$$

for each $\omega \in \Omega$. Suppose that $S$ is a continuous random operator, then

$$
S\left(\omega, S\left(\omega, \eta_{2 n}(\omega)\right)\right) \longrightarrow S(\omega, \xi(\omega)), \quad S\left(\omega, A\left(\omega, \eta_{2 n-2}(\omega)\right)\right) \longrightarrow S(\omega, \xi(\omega))
$$

for every $\omega \in \Omega$. Since $A$ and $S$ are compatible random operators, this gives

$$
A\left(\omega, S\left(\omega, \eta_{2 n-2}(\omega)\right)\right) \longrightarrow S(\omega, \xi(\omega))=h(\omega) \quad \text { for each } \omega \in \Omega
$$

when $n \rightarrow \infty$. Since $X$ is a complete separable metric space, so for any continuous random operator $S$, a mapping $h: \Omega \rightarrow X$ defined by $h(\omega)=S(\omega, \xi(\omega))$ is measurable $[15,28]$. Since $A(\omega, X) \subset T(\omega, X)$ for each $\omega \in \Omega$, define mappings $\zeta_{2 n-2}: \Omega \rightarrow X$ as $A(\omega, S(\omega$, $\left.\left.\eta_{2 n-2}(\omega)\right)\right)=T\left(\omega, \zeta_{2 n-2}(\omega)\right)$ for each $\omega \in \Omega$. Thus

$$
T\left(\omega, \zeta_{2 n-2}(\omega)\right) \longrightarrow h(\omega), \quad S\left(\omega, S\left(\omega, \eta_{2 n}(\omega)\right)\right) \longrightarrow h(\omega)
$$


for every $\omega \in \Omega$. Since

$$
\begin{aligned}
& d\left(A\left(\omega, S\left(\omega, \eta_{2 n-2}(\omega)\right)\right), B\left(\omega, \zeta_{2 n-2}(\omega)\right)\right) \\
& \quad \leq k(\omega) d\left(S\left(\omega, S\left(\omega, \eta_{2 n}(\omega)\right)\right), T\left(\omega, \zeta_{2 n-2}(\omega)\right)\right)
\end{aligned}
$$

for each $\omega \in \Omega$, on taking limit $n \rightarrow \infty$, we have $\left.B\left(\omega, \zeta_{2 n-2}(\omega)\right)\right) \rightarrow h(\omega)$ for each $\omega \in \Omega$. Also

$$
\begin{aligned}
& d\left(A(\omega, \xi(\omega)), B\left(\omega, \zeta_{2 n-2}(\omega)\right)\right) \\
& \quad \leq k(\omega) d\left(S(\omega, \xi(\omega)), T\left(\omega, \zeta_{2 n-2}(\omega)\right)\right) .
\end{aligned}
$$

Applying limit $n \rightarrow \infty$, we have $d(A(\omega, \xi(\omega)), h(\omega)) \leq k(\omega) d(S(\omega, \xi(\omega)), h(\omega))$. So $A(\omega$, $\xi(\omega))=h(\omega)$ for each $\omega \in \Omega$. Now $A(\omega, X) \subset T(\omega, X)$ and $A(\omega, \xi(\omega)) \in A(\omega, X)$ for each $\omega \in \Omega$ allow to define the measurable mapping $\rho_{0}: \Omega \rightarrow X$ as $A(\omega, \xi(\omega))=T\left(\omega, \rho_{0}(\omega)\right)$. Using (1) we have $A(\omega, \xi(\omega))=B\left(\omega, \rho_{0}(\omega)\right)$ for each $\omega \in \Omega$. So

$$
h(\omega)=A(\omega, \xi(\omega))=B\left(\omega, \rho_{0}(\omega)\right)=T\left(\omega, \rho_{0}(\omega)\right)=S(\omega, \xi(\omega))
$$

for every $\omega \in \Omega$. Thus $A(\omega, S(\omega, \xi(\omega)))=S(\omega, A(\omega, \xi(\omega)))$ gives

$$
A(\omega, h(\omega))=S(\omega, h(\omega)) \quad \text { for every } \omega \in \Omega
$$

Moreover

$$
A(\omega, A(\omega, \xi(\omega)))=A(\omega, S(\omega, \xi(\omega)))=S(\omega, A(\omega, \xi(\omega)))=S(\omega, S(\omega, \xi(\omega)))
$$

for each $\omega \in \Omega$. Also

$$
B\left(\omega, B\left(\omega, \rho_{0}(\omega)\right)\right)=B\left(\omega, T\left(\omega, \rho_{0}(\omega)\right)\right)=T\left(\omega, B\left(\omega, \rho_{0}(\omega)\right)\right)=T\left(\omega, T\left(\omega, \rho_{0}(\omega)\right)\right)
$$

for each $\omega \in \Omega$. Using (2.30) we have

$$
\begin{aligned}
& A(\omega, h(\omega))=A(\omega, A(\omega, \xi(\omega)))=A(\omega, h(\omega))=h(\omega), \\
& B\left(\omega, \rho_{0}(\omega)\right)=B\left(\omega, B\left(\omega, \rho_{0}(\omega)\right)\right)=B(\omega, h(\omega))=h(\omega),
\end{aligned}
$$

for each $\omega \in \Omega$. So, $h$ is a random fixed point of $A, B, S$, and $T$. Uniqueness of random fixed point follows from (1).

\section{Common random fixed points in symmetric spaces}

It is interesting to note that certain theorems in metric spaces can be proved without using some of the defining properties of metric. Hicks [14] exploiting this fact, established some common fixed point theorems in symmetric spaces. In this section, we obtain common random fixed point of two and four random operators under generalized contractive condition in symmetric spaces. 
Theorem 3.1. Let $(X, d)$ be a separable symmetric space that satisfies (w.1) and $\left(H_{E}\right)$. Let $T, S: \Omega \times X \rightarrow X$ be two weakly compatible random operators satisfying the property $(I)$. Moreover, for all $x, y \in X$ we have

$$
\begin{aligned}
& d(T(\omega, x), T(\omega, y)) \\
& \quad \leq \phi(\max \{d(S(\omega, x), S(\omega, y)), d(S(\omega, x), T(\omega, y)), d(T(\omega, y), S(\omega, y))\})
\end{aligned}
$$

for every $\omega \in \Omega$. If $T(\omega, X) \subset S(\omega, X)$ and one of $T(\omega, X)$ or $S(\omega, X)$ is a complete subspace of $X$ for every $\omega \in \Omega$, then $T$ and $S$ have unique random fixed point.

Proof. Since random operators $T$ and $S$ satisfy the property $(I)$, so there exists a sequence $\left\{\xi_{n}\right\}$ in $M(\Omega, X)$ such that

$$
\lim _{n \rightarrow \infty} d\left(T\left(\omega, \xi_{n}(\omega)\right), \xi(\omega)\right)=\lim _{n \rightarrow \infty} d\left(S\left(\omega, \xi_{n}(\omega)\right), \xi(\omega)\right)=0
$$

for every $\omega \in \Omega$, for some $\xi \in M(\Omega, X)$. Therefore by property $\left(H_{E}\right)$, we have $\lim _{n \rightarrow \infty} d\left(T\left(\omega, \xi_{n}(\omega)\right), S\left(\omega, \xi_{n}(\omega)\right)\right)=0$ for every $\omega \in \Omega$. Suppose $S(\omega, X)$ is a complete subspace of $X$ for every $\omega \in \Omega$. Let $\xi_{1}: \Omega \rightarrow X$ be the limit of the sequence of measurable mappings $\left\{S\left(\omega, \xi_{n}(\omega)\right)\right\}$ and $S\left(\omega, \xi_{n}(\omega)\right) \in S(\omega, X)$ for every $\omega \in \Omega$ and $n \in \mathbb{N}$. Now since $X$ is separable, therefore $\xi_{1} \in M(\Omega, X)$. Moreover $\xi_{1}(\omega) \in S(\omega, X)$ for every $\omega \in \Omega$. This allows obtaining the measurable mapping $\bar{\xi}: \Omega \rightarrow X$ such that $\xi(\omega)=S(\omega, \bar{\xi}(\omega))$. Now we show that $T(\omega, \bar{\xi}(\omega))=S(\omega, \bar{\xi}(\omega))$ for every $\omega \in \Omega$. Consider

$$
\begin{gathered}
d\left(T(\omega, \bar{\xi}(\omega)), T\left(\omega, \xi_{n}(\omega)\right)\right) \\
\leq \phi\left(\operatorname { m a x } \left\{d\left(S(\omega, \bar{\xi}(\omega)), S\left(\omega, \xi_{n}(\omega)\right)\right), d\left(S(\omega, \bar{\xi}(\omega)), T\left(\omega, \xi_{n}(\omega)\right)\right),\right.\right. \\
\left.\left.d\left(S\left(\omega, \xi_{n}(\omega)\right), T\left(\omega, \xi_{n}(\omega)\right)\right)\right\}\right) \\
<\max \left\{d\left(\xi(\omega), S\left(\omega, \xi_{n}(\omega)\right)\right), d\left(\xi(\omega), T\left(\omega, \xi_{n}(\omega)\right)\right)\right. \\
\left.d\left(S\left(\omega, \xi_{n}(\omega)\right), T\left(\omega, \xi_{n}(\omega)\right)\right)\right\} \quad \text { for every } \omega \in \Omega .
\end{gathered}
$$

Taking $n \rightarrow \infty$, we have $d\left(T(\omega, \bar{\xi}(\omega)), T\left(\omega, \xi_{n}(\omega)\right)\right) \rightarrow 0$ for every $\omega \in \Omega$. Thus by (w.1) we have $T(\omega, \bar{\xi}(\omega))=S(\omega, \bar{\xi}(\omega))$ for every $\omega \in \Omega$. The weak compatibility of random mappings $T$ and $S$ implies that $T(\omega, S(\omega, \bar{\xi}(\omega)))=S(\omega, T(\omega, \bar{\xi}(\omega)))$, then $T(\omega, T(\omega, \bar{\xi}(\omega)))=$ $T(\omega, S(\omega, \bar{\xi}(\omega)))=S(\omega, T(\omega, \bar{\xi}(\omega)))=S(\omega, S(\omega, \bar{\xi}(\omega)))$ for every $\omega \in \Omega$. Let us show that $T(\omega, T(\omega, \bar{\xi}(\omega)))=T(\omega, \bar{\xi}(\omega))$ for each $\omega \in \Omega$. If not, then for some $\omega \in \Omega$, consider

$$
\begin{aligned}
& d(T(\omega, \bar{\xi}(\omega)), T(\omega, \xi(\omega))) \\
& \leq \phi(\max \{d(S(\omega, \bar{\xi}(\omega)), S(\omega, \xi(\omega))), d(S(\omega, \bar{\xi}(\omega)), T(\omega, \xi(\omega))) \\
&d(S(\omega, \xi(\omega)), T(\omega, \xi(\omega)))\}) \\
& \leq \phi(\max \{d(T(\omega, \bar{\xi}(\omega)), T(\omega, \xi(\omega))), d(T(\omega, \bar{\xi}(\omega)), T(\omega, \xi(\omega)))\}) \\
& \leq \phi(d(T(\omega, \bar{\xi}(\omega)), T(\omega, \xi(\omega)))) \\
&< d(T(\omega, \bar{\xi}(\omega)), T(\omega, \xi(\omega)))
\end{aligned}
$$


which is a contradiction, so $T(\omega, \bar{\xi}(\omega))$ is a random fixed point of $T$. Now $T(\omega, \bar{\xi}(\omega))=$ $T(\omega, T(\omega, \bar{\xi}(\omega)))=S(\omega, T(\omega, \bar{\xi}(\omega)))$ for every $\omega \in \Omega$. Therefore $T(\omega, \bar{\xi}(\omega))$ is a common random fixed point of $T$ and $S$. The proof is similar when $T(\omega, X)$ is supposed to be a complete subspace of $X$ for every $\omega \in \Omega$, as $T(\omega, X) \subset S(\omega, X)$ for each $\omega \in \Omega$. To prove the uniqueness of common random fixed point, let $\eta, \bar{\eta}: \Omega \rightarrow X$ be two common random fixed points of random operators $T$ and $S$ such that $\eta(\omega) \neq \bar{\eta}(\omega)$ for some $\omega \in \Omega$. Consider

$$
\begin{aligned}
d(\bar{\eta}(\omega), \eta(\omega))= & d(T(\omega, \bar{\eta}(\omega)), T(\omega, \eta(\omega))) \\
\leq & \phi(\max \{d(S(\omega, \bar{\eta}(\omega)), S(\omega, \eta(\omega))), d(S(\omega, \bar{\eta}(\omega)), T(\omega, \eta(\omega))), \\
& \quad d(S(\omega, \bar{\eta}(\omega)), T(\omega, \bar{\eta}(\omega)))\}) \\
\leq & \phi(d(\bar{\eta}(\omega), \eta(\omega)))<d(\bar{\eta}(\omega), \eta(\omega)) .
\end{aligned}
$$

This contradiction shows $\eta(\omega)=\bar{\eta}(\omega)$ for every $\omega \in \Omega$.

Theorem 3.2. Let $(X, d)$ be a separable symmetric space that satisfies (w.1), (w.2), and $\left(H_{E}\right)$. Let $(A, T)$ and $(B, S)$ be two pairs of weakly compatible random operators from $\Omega \times X$ to $X$ such that one of the pairs $(A, S)$ or $(B, T)$ satisfies the property $(I)$. Moreover

$$
\begin{aligned}
& d(A(\omega, x), B(\omega, y)) \\
& \quad \leq \phi(\max \{d(S(\omega, x), T(\omega, y)), d(S(\omega, x), B(\omega, y)), d(T(\omega, y), B(\omega, y))\})
\end{aligned}
$$

for every $\omega \in \Omega$. If $B(\omega, X) \subset S(\omega, X), A(\omega, X) \subset T(\omega, X)$, and one of $T(\omega, X), S(\omega, X)$, $B(\omega, X)$, or $S(\omega, X)$ is complete subspace of $X$ for every $\omega \in \Omega$, then $A, B, T$, and $S$ have unique common random fixed point.

Proof. Suppose the pair $(B, T)$ of random mappings satisfies the property $(I)$. So there exists a sequence $\left\{\xi_{n}\right\}$ in $M(\Omega, X)$ such that

$$
\lim _{n \rightarrow \infty} d\left(B\left(\omega, \xi_{n}(\omega)\right), \xi(\omega)\right)=\lim _{n \rightarrow \infty} d\left(T\left(\omega, \xi_{n}(\omega)\right), \xi(\omega)\right)=0
$$

for every $\omega \in \Omega$ for some $\xi \in M(\Omega, X)$. As $\left\{B\left(\omega, \xi_{n}(\omega)\right)\right\}$ is a sequence of measurable mappings and $B\left(\omega, \xi_{n}(\omega)\right) \in B(\omega, X)$ for every $\omega \in \Omega$ and $n \in \mathbb{N}$, now the fact $B(\omega, X) \subset$ $S(\omega, X)$ allows obtaining the sequence of measurable mappings $\eta_{n}: \Omega \rightarrow X$ such that $B\left(\omega, \xi_{n}(\omega)\right)=S\left(\omega, \eta_{n}(\omega)\right)$ for every $\omega \in \Omega$. Hence, $\lim _{n \rightarrow \infty} d\left(S\left(\omega, \eta_{n}(\omega)\right), \xi(\omega)\right)=0$ for every $\omega \in \Omega$. Now we show that $\lim _{n \rightarrow \infty} d\left(T\left(\omega, \eta_{n}(\omega)\right), \xi(\omega)\right)=0$ for every $\omega \in \Omega$. For this consider

$$
\begin{aligned}
d\left(A\left(\omega, \eta_{n}(\omega)\right), B\left(\omega, \xi_{n}(\omega)\right)\right) & \\
\leq & \phi\left(\operatorname { m a x } \left\{d\left(S\left(\omega, \eta_{n}(\omega)\right), T\left(\omega, \xi_{n}(\omega)\right)\right), d\left(S\left(\omega, \eta_{n}(\omega)\right), B\left(\omega, \xi_{n}(\omega)\right)\right),\right.\right. \\
& \left.\left.d\left(T\left(\omega, \xi_{n}(\omega)\right), B\left(\omega, \xi_{n}(\omega)\right)\right)\right\}\right) \\
\leq & \phi\left(\max \left\{d\left(B\left(\omega, \xi_{n}(\omega)\right), T\left(\omega, \xi_{n}(\omega)\right)\right), d\left(T\left(\omega, \xi_{n}(\omega)\right), B\left(\omega, \xi_{n}(\omega)\right)\right)\right\}\right) \\
\leq & \phi\left(d\left(B\left(\omega, \xi_{n}(\omega)\right), T\left(\omega, \xi_{n}(\omega)\right)\right)\right) \\
< & d\left(B\left(\omega, \xi_{n}(\omega)\right), T\left(\omega, \xi_{n}(\omega)\right)\right)
\end{aligned}
$$


12 Common random fixed points of compatible random operators

for every $\omega \in \Omega$. Therefore by property $\left(H_{E}\right)$, we have

$$
\lim _{n \rightarrow \infty} d\left(B\left(\omega, \xi_{n}(\omega)\right), T\left(\omega, \xi_{n}(\omega)\right)\right)=0 \quad \text { for every } \omega \in \Omega
$$

Hence,

$$
\lim _{n \rightarrow \infty} d\left(B\left(\omega, \xi_{n}(\omega)\right), A\left(\omega, \eta_{n}(\omega)\right)\right)=0 \quad \text { for every } \omega \in \Omega
$$

By (w.2), we deduce that $\lim _{n \rightarrow \infty} d\left(A\left(\omega, \eta_{n}(\omega)\right), \xi(\omega)\right)=0$, for every $\omega \in \Omega$. Suppose for every $\omega \in \Omega, S(\omega, X)$ is a complete subspace of $X$. Now $\left\{S\left(\omega, \eta_{n}(\omega)\right\}\right.$ is a sequence of measurable mappings and $S\left(\omega, \eta_{n}(\omega)\right) \in S(\omega, X)$ for every $\omega \in \Omega$. Let $\xi_{1}: \Omega \rightarrow X$ be the limit of the sequence of measurable mappings $\left\{S\left(\omega, \xi_{n}(\omega)\right)\right\}$. Since $X$ is separable, therefore $\xi_{1} \in M(\Omega, X)$. Moreover $\xi_{1}(\omega) \in S(\omega, X)$ for every $\omega \in \Omega$. This allows obtaining the measurable mapping $\bar{\xi}: \Omega \rightarrow X$ such that $\xi(\omega)=S(\omega, \bar{\xi}(\omega))$. Now consider

$$
\begin{aligned}
\lim _{n \rightarrow \infty} d\left(A\left(\omega, \eta_{n}(\omega)\right), S(\omega, \bar{\xi}(\omega))\right) & =\lim _{n \rightarrow \infty} d\left(B\left(\omega, \xi_{n}(\omega)\right), S(\omega, \bar{\xi}(\omega))\right) \\
& =\lim _{n \rightarrow \infty} d\left(T\left(\omega, \xi_{n}(\omega)\right), S(\omega, \bar{\xi}(\omega))\right) \\
& =\lim _{n \rightarrow \infty} d\left(S\left(\omega, \eta_{n}(\omega)\right), S(\omega, \bar{\xi}(\omega))\right)=0
\end{aligned}
$$

for every $\omega \in \Omega$. Thus

$$
\begin{aligned}
& d\left(A(\omega, \bar{\xi}(\omega)), B\left(\omega, \xi_{n}(\omega)\right)\right) \\
& \leq \phi(\max \{ d\left(S(\omega, \bar{\xi}(\omega)), T\left(\omega, \xi_{n}(\omega)\right)\right), d\left(S(\omega, \bar{\xi}(\omega)), B\left(\omega, \xi_{n}(\omega)\right)\right) \\
&\left.\left.d\left(T\left(\omega, \xi_{n}(\omega)\right), B\left(\omega, \xi_{n}(\omega)\right)\right)\right\}\right)
\end{aligned}
$$

for each $\omega \in \Omega$. This immediately gives

$$
\lim _{n \rightarrow \infty} d\left(A(\omega, \bar{\xi}(\omega)), B\left(\omega, \xi_{n}(\omega)\right)\right)=0 \quad \text { for every } \omega \in \Omega
$$

By (w.1), we have $A(\omega, \bar{\xi}(\omega))=S(\omega, \bar{\xi}(\omega))$ for every $\omega \in \Omega$. The weak compatibility of random operators $A$ and $S$ implies that $S(\omega, A(\omega, \bar{\xi}(\omega)))=A(\omega, S(\omega, \bar{\xi}(\omega)))$ for every $\omega \in$ $\Omega$. Now

$$
A(\omega, A(\omega, \bar{\xi}(\omega)))=A(\omega, S(\omega, \bar{\xi}(\omega)))=S(\omega, A(\omega, \bar{\xi}(\omega)))=S(\omega, S(\omega, \bar{\xi}(\omega)))
$$

for every $\omega \in \Omega$. As $A(\omega, \bar{\xi}(\omega)) \in A(\omega, X)$ for every $\omega \in \Omega$ where $\bar{\xi} \in M(\Omega, X)$, the assumption $A(\omega, X) \subset T(\omega, X)$ for every $\omega \in \Omega$ allows obtaining $\bar{\xi}_{1} \in M(\Omega, X)$ such that 
$A(\omega, \bar{\xi}(\omega))=T\left(\omega, \bar{\xi}_{1}(\omega)\right)$ for every $\omega \in \Omega$. We now show that for every $\omega \in \Omega, B\left(\omega, \bar{\xi}_{1}(\omega)\right)$ $=T\left(\omega, \bar{\xi}_{1}(\omega)\right)$. If not, then for some $\omega \in \Omega$, consider

$$
\begin{aligned}
& d\left(A(\omega, \bar{\xi}(\omega)), B\left(\omega, \bar{\xi}_{1}(\omega)\right)\right) \\
& \leq \phi\left(\operatorname { m a x } \left\{d\left(S(\omega, \bar{\xi}(\omega)), T\left(\omega, \bar{\xi}_{1}(\omega)\right)\right), d\left(S(\omega, \bar{\xi}(\omega)), B\left(\omega, \bar{\xi}_{1}(\omega)\right)\right)\right.\right. \\
&\left.\left.\quad d\left(T\left(\omega, \bar{\xi}_{1}(\omega)\right), B\left(\omega, \bar{\xi}_{1}(\omega)\right)\right)\right\}\right) \\
& \leq \phi\left(\max \left\{d\left(A(\omega, \bar{\xi}(\omega)), B\left(\omega, \bar{\xi}_{1}(\omega)\right)\right), d\left(A(\omega, \bar{\xi}(\omega)), B\left(\omega, \bar{\xi}_{1}(\omega)\right)\right)\right\}\right) \\
& \leq \phi\left(d\left(A(\omega, \bar{\xi}(\omega)), B\left(\omega, \bar{\xi}_{1}(\omega)\right)\right)\right)<d\left(A(\omega, \bar{\xi}(\omega)), B\left(\omega, \bar{\xi}_{1}(\omega)\right)\right)
\end{aligned}
$$

which is a contradiction. Hence,

$$
B\left(\omega, \bar{\xi}_{1}(\omega)\right)=T\left(\omega, \bar{\xi}_{1}(\omega)\right)=A(\omega, \bar{\xi}(\omega))=S(\omega, \bar{\xi}(\omega)) \quad \text { for every } \omega \in \Omega
$$

The weak compatibility of random operators $B$ and $T$ implies that

$$
\begin{gathered}
B\left(\omega, T\left(\omega, \bar{\xi}_{1}(\omega)\right)\right)=T\left(\omega, B\left(\omega, \bar{\xi}_{1}(\omega)\right)\right) \quad \text { for every } \omega \in \Omega, \\
T\left(\omega, T\left(\omega, \bar{\xi}_{1}(\omega)\right)\right)=T\left(\omega, B\left(\omega, \bar{\xi}_{1}(\omega)\right)\right)=B\left(\omega, T\left(\omega, \bar{\xi}_{1}(\omega)\right)\right)=B\left(\omega, B\left(\omega, \bar{\xi}_{1}(\omega)\right)\right)
\end{gathered}
$$

for each $\omega \in \Omega$. Let us show that $A(\omega, A(\omega, \bar{\xi}(\omega)))=A(\omega, \bar{\xi}(\omega))$ for each $\omega \in \Omega$. If not, then for some $\omega \in \Omega$, consider

$$
\begin{aligned}
& d(A(\omega, \bar{\xi}(\omega)), A(\omega, A(\omega, \bar{\xi}(\omega)))) \\
&= d\left(A(\omega, A(\omega, \bar{\xi}(\omega))), B\left(\omega, \bar{\xi}_{1}(\omega)\right)\right) \\
& \leq \phi\left(\operatorname { m a x } \left\{d\left(S(\omega, A(\omega, \bar{\xi}(\omega))), T\left(\omega, \bar{\xi}_{1}(\omega)\right)\right), d\left(S(\omega, A(\omega, \bar{\xi}(\omega))), B\left(\omega, \bar{\xi}_{1}(\omega)\right)\right),\right.\right. \\
&\left.\left.\quad d\left(T\left(\omega, \bar{\xi}_{1}(\omega)\right), B\left(\omega, \bar{\xi}_{1}(\omega)\right)\right)\right\}\right) \\
& \leq\phi(\max \{d(A(\omega, A(\omega, \bar{\xi}(\omega))), A(\omega, \bar{\xi}(\omega))), A(\omega, A(\omega, \bar{\xi}(\omega))), A(\omega, \bar{\xi}(\omega)))\}) \\
& \leq \phi(d(A(\omega, A(\omega, \bar{\xi}(\omega))), A(\omega, \bar{\xi}(\omega)))) \\
&< d(A(\omega, A(\omega, \bar{\xi}(\omega))), A(\omega, \bar{\xi}(\omega))),
\end{aligned}
$$

which is a contradiction. Therefore

$$
A(\omega, A(\omega, \bar{\xi}(\omega)))=A(\omega, \bar{\xi}(\omega))=S(\omega, A(\omega, \bar{\xi}(\omega))) \quad \text { for every } \omega \in \Omega
$$

So $A(\omega, \bar{\xi}(\omega))$ is common random fixed point of random operators $A$ and S. Similarly, $B\left(\omega, \bar{\xi}_{1}(\omega)\right)$ is common random fixed point of random operators $B$ and T. Since $A(\omega$, $\bar{\xi}(\omega))=B\left(\omega, \bar{\xi}_{1}(\omega)\right)$ for every $\omega \in \Omega$, thus $A(\omega, \bar{\xi}(\omega))$ is common random fixed point of random operators $A, B, S$, and $T$. The proof is similar when for every $\omega \in \Omega, T(\omega, X)$ is a complete subspace of $X$. The cases in which $A(\omega, X)$ or $B(\omega, X)$ is a complete subspace of $X$ for every $\omega \in \Omega$ are similar to the cases in which $T(\omega, X)$ or $S(\omega, X)$, respectively, 
is a complete subspace of $X$, since $A(\omega, X) \subset T(\omega, X)$ and $B(\omega, X) \subset S(\omega, X)$ for every $\omega \in \Omega$. To establish the uniqueness of common random fixed point of random operators, let $\xi$ and $\eta$ be two common random fixed points of the random operators such that $\xi(\omega) \neq \eta(\omega)$ for some $\omega \in \Omega$. Consider

$$
\begin{aligned}
d(\xi(\omega), \eta(\omega))= & d(A(\omega, \xi(\omega)), B(\omega, \eta(\omega))) \\
\leq & \phi(\max \{d(S(\omega, \xi(\omega)), T(\omega, \eta(\omega))), d(S(\omega, \xi(\omega)), B(\omega, \eta(\omega))) \\
& d(T(\omega, \eta(\omega)), B(\omega, \eta(\omega)))\}) \\
\leq & \phi(d(\xi(\omega), \eta(\omega)))<d(\xi(\omega), \eta(\omega)),
\end{aligned}
$$

which is a contradiction. So the result follows.

\section{Acknowledgment}

Authors are thankful to the referee for his critical remarks to improve the presentation of the paper.

\section{References}

[1] R. P. Agarwal, M. Meehan, and D. O’Regan, Fixed Point Theory and Applications, Cambridge Tracts in Mathematics, vol. 141, Cambridge University Press, Cambridge, 2001.

[2] J.-P. Aubin and H. Frankowska, Set-Valued Analysis, Systems \& Control: Foundations \& Applications, vol. 2, Birkhäuser Boston, Massachusetts, 1990.

[3] I. Beg, Random fixed points of random operators satisfying semicontractivity conditions, Mathematica Japonica 46 (1997), no. 1, 151-155.

[4] _ Approximation of random fixed points in normed spaces, Nonlinear Analysis 51 (2002), no. $8,1363-1372$.

[5] I. Beg and A. Azam, Fixed points of asymptotically regular multivalued mappings, Journal of Australian Mathematical Society. Series A 53 (1992), no. 3, 313-326.

[6] I. Beg and N. Shahzad, Random fixed points of random multivalued operators on Polish spaces, Nonlinear Analysis 20 (1993), no. 7, 835-847.

[7] _ Random fixed point theorems for nonexpansive and contractive-type random operators on Banach spaces, Journal of Applied Mathematics and Stochastic Analysis 7 (1994), no. 4, 569580.

[8] _ Common random fixed points of random multivalued operators on metric spaces, Bollettino della Unione Matemàtica Italiana, Serie VII. A 9 (1995), no. 3, 493-503.

[9] A. T. Bharucha-Reid, Random Integral Equations, Academic Press, New York, 1972.

[10] - Fixed point theorems in probabilistic analysis, Bulletin of the American Mathematical Society 82 (1976), no. 5, 641-657.

[11] G. Górnowicz, Topological Fixed Point Theory of Multivalued Mappings, Mathematics and Its Applications, vol. 495, Kluwer Academic, Dordrechit, 1999.

[12] O. Hanš, Reduzierende zufällige Transformationen, Czechoslovak Mathematical Journal 7(82) (1957), 154-158.

[13] _ Random operator equations, Proceedings of 4th Berkeley Symposium on Mathematical Statistics and Probability, Vol. II, Part I, University of California Press, California, 1961, pp. 185202.

[14] T. L. Hicks and B. E. Rhoades, Fixed point theory in symmetric spaces with applications to probabilistic spaces, Nonlinear Analysis 36 (1999), no. 3, 331-344.

[15] C. J. Himmelberg, Measurable relations, Fundamenta Mathematicae 87 (1975), 53-72. 
[16] S. Itoh, Random fixed-point theorems with an application to random differential equations in $\mathrm{Ba}$ nach spaces, Journal of Mathematical Analysis and Applications 67 (1979), no. 2, 261-273.

[17] G. Jungck, Common fixed points for commuting and compatible maps on compacta, Proceedings of the American Mathematical Society 103 (1988), no. 3, 977-983.

[18] A. Meir and E. Keeler, A theorem on contraction mappings, Journal of Mathematical Analysis and Applications 28 (1969), 326-329.

[19] D. O'Regan, Fixed points and random fixed points for weakly inward approximable maps, Proceedings of the American Mathematical Society 126 (1998), no. 10, 3045-3053.

[20] R. P. Pant, Common fixed points of noncommuting mappings, Journal of Mathematical Analysis and Applications 188 (1994), no. 2, 436-440.

[21] Common fixed point theorems for contractive maps, Journal of Mathematical Analysis and Applications 226 (1998), no. 1, 251-258.

[22] - Meir-Keeler type fixed point theorems and dynamics of functions, Demonstratio Mathematica 36 (2003), no. 1, 199-206.

[23] N. S. Papageorgiou, Random fixed point theorems for measurable multifunctions in Banach spaces, Proceedings of the American Mathematical Society 97 (1986), no. 3, 507-514.

[24] S. Park and J. S. Bae, Extensions of a fixed point theorem of Meir and Keeler, Arkiv för Matematik 19 (1981), no. 2, 223-228.

[25] V. M. Sehgal and S. P. Singh, On random approximations and a random fixed point theorem for set valued mappings, Proceedings of the American Mathematical Society 95 (1985), no. 1, 91-94.

[26] A. Špaček, Zufällige gleichungen, Czechoslovak Mathematical Journal 5(80) (1955), 462-466.

[27] K.-K. Tan and X.-Z. Yuan, On deterministic and random fixed points, Proceedings of the American Mathematical Society 119 (1993), no. 3, 849-856.

[28] D. H. Wagner, Survey of measurable selection theorems, SIAM Journal on Control and Optimization 15 (1977), no. 5, 859-903.

[29] W. A. Wilson, On semi-metric spaces, American Journal of Mathematics 53 (1931), no. 2, 361373.

Ismat Beg: Department of Mathematics and Center for Advanced Studies in Mathematics, Lahore University of Management Sciences, 54792 Lahore, Pakistan

E-mail address: ibeg@lums.edu.pk

Mujahid Abbas: Department of Mathematics and Center for Advanced Studies in Mathematics, Lahore University of Management Sciences, 54792 Lahore, Pakistan

E-mail address: mujahid@lums.edu.pk 


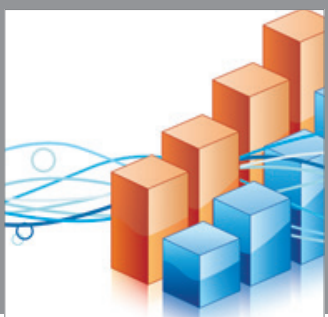

Advances in

Operations Research

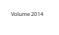

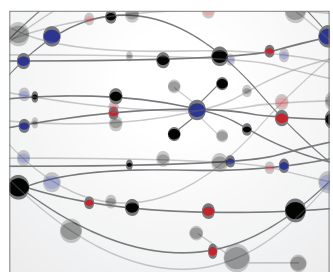

\section{The Scientific} World Journal
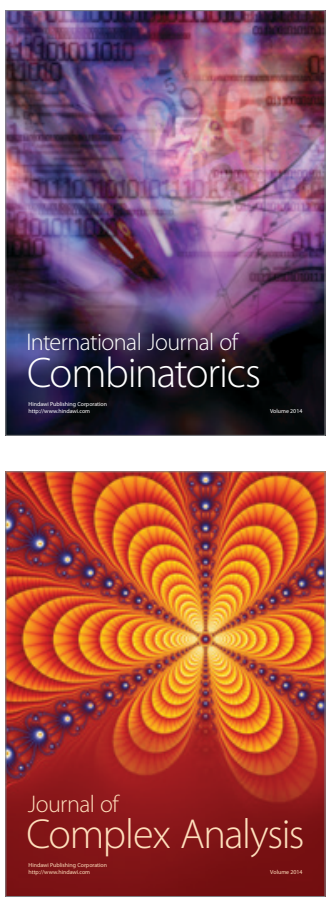

International Journal of

Mathematics and

Mathematical

Sciences
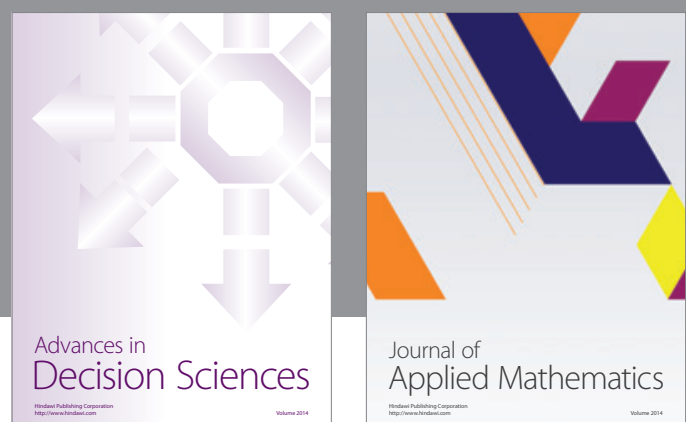

Journal of

Applied Mathematics
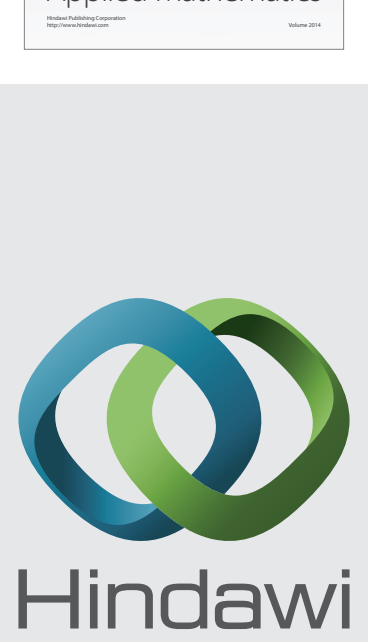

Submit your manuscripts at http://www.hindawi.com
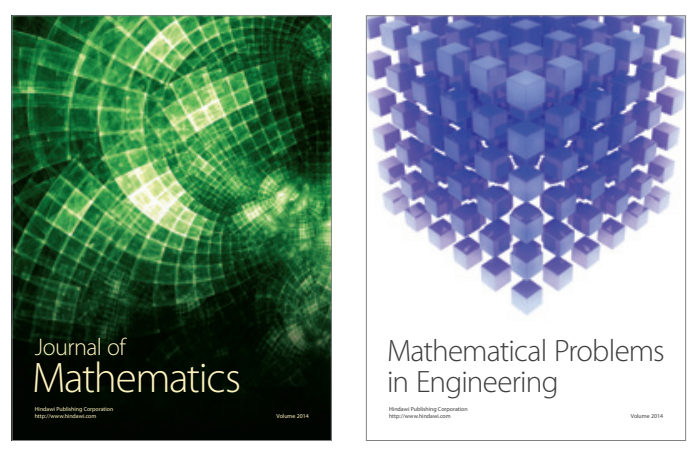

Mathematical Problems in Engineering
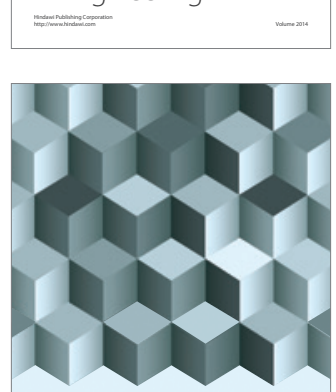

Journal of

Function Spaces
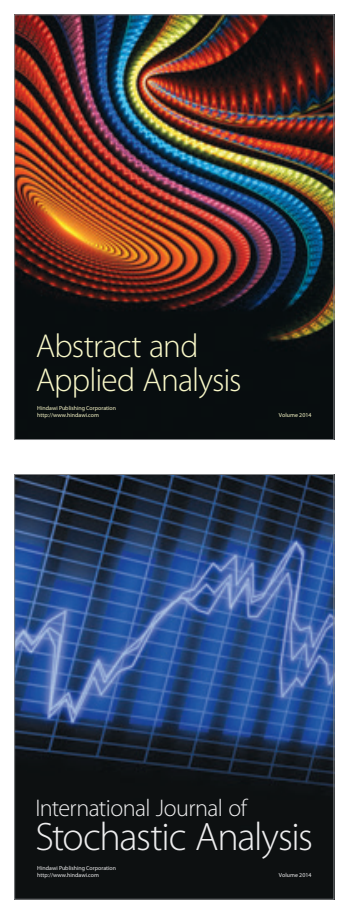

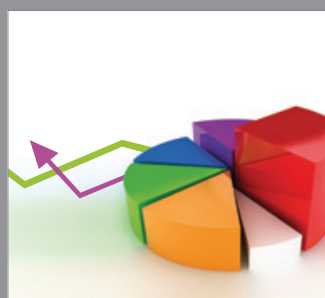

ournal of

Probability and Statistics

Promensencen
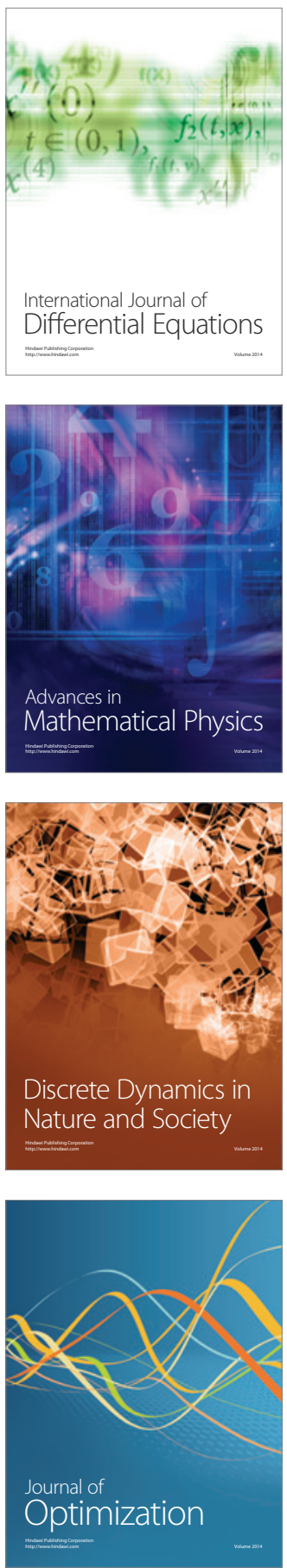